Resistance to DSCG in acute asthma is greater therefore, than to salbutamol. However, clinical observation suggests that resistance to beta-2 stimulants does also increase with severity of the attack. It would appear probable that only when severity exceeds the point at which measurements can be made with a peak flow meter does resistance to salbutamol become pronounced. Although placebo effects are greater in the attack than between attacks, again no information is available about attacks severe enough to prevent measurement using a peak flow meter.

This work was supported by grants from the Intensive Care Fund, Alder Hey Children's Hospital, and Allen \& Hanburys Limited.
References

1 Chung J T N, Jones R S. Bronchodilator effect of sodium cromoglycate and its clinical implications. $\mathrm{Br}$ Med $\mathrm{J}$ 1979; ii: $1033-4$.

2 Lawford P, Jones B J M, Milledge J S. Comparison of intravenous and nebulised salbutamol in initial treatment of severe asthma. Br Med J 1978; i: 84.

3 Anonymous. Intravenous versus inhaled salbutamol. Lancet 1978 ; i: 80.

4 Williams S, Seaton A. Intravenous or inhaled salbutamol in severe acute asthma. Thorax $1977 ; 32$ : 555-8.

Correspondence to Dr R S Jones, Respiratory Unit, Alder Hey Children's Hospital, Eaton Road, Liverpool L12 2AP.

Received 16 February 1981

\title{
The steatocrit: a simple method for estimating stool fat content in newborn infants
}

\author{
P PHUAPRADIT, A NARANG, P MENDONCA, D A HARRIS, AND J D BAUM \\ University Department of Paediatrics John Radcliffe Hospital, Oxford
}

SUMmARY A simple micromethod is described for estimating the stool fat content of newborn babies. It is quick and reliable, requires only a small amount of stool, and can be used as a screening test for newborn babies suspected of fat malabsorption.

Many infants, especially those of low birthweight or short gestation, gain weight slowly in the early neonatal period. There are many reasons for such poor weight gain among which the loss of excess dietary fat in the stools needs to be considered. We have previously shown that it is possible to use a very simple method based on microcentrifugation for predicting the fat content and caloric value of milk. ${ }^{1}$ In this study we have investigated the application of microcentrifugation to newborn infants' stool samples to measure stool fat content.

\section{Method}

Water content of the stool. In order to express the stool fat per gram wet weight of stool, it was first necessary to ensure that the water content of the stool was not in itself an important variable. Ten samples of stool from 3 term and 7 preterm infants,
6 fed on expressed breast milk and 4 on a milk formula, were weighed wet and then freeze dried for 24 hours and reweighed. The water content of the stool was thus estimated and expressed as a percentage of the wet weight.

Stool fat measurement. Stools were weighed and homogenised with a small amount of sand $(0.06 \mathrm{~g})$ and two volumes of water, using a pestle and mortar and a hand homogeniser. Small amounts of well homogenised stool (about $75 \mu \mathrm{l}$ ) were drawn by a pipette into a capillary tube (for this technique blood pH capillary tubes cut to the same length as standard glass capillary tubes were used). They were sealed at one end by a flame and centrifuged for 15 minutes at $12000 \mathrm{~g}$, using a haematocrit centrifuge (Hawksley, London).

After centrifugation the tubes were removed immediately and placed vertically. The length of the fat layer at the top and that of the solid layer at the bottom was measured with vernier callipers to the nearest $0.05 \mathrm{~mm}$. Stool fat content was expressed as a percentage (steatocrit) of the total length of the solid column in the tube (that is fat layer + solid layer) (Fig. 1). The steatocrit was measured in duplicate. The stool fat content was also measured by Sobel's method $^{2}$ which requires $3 \mathrm{~g}$ of stool. 


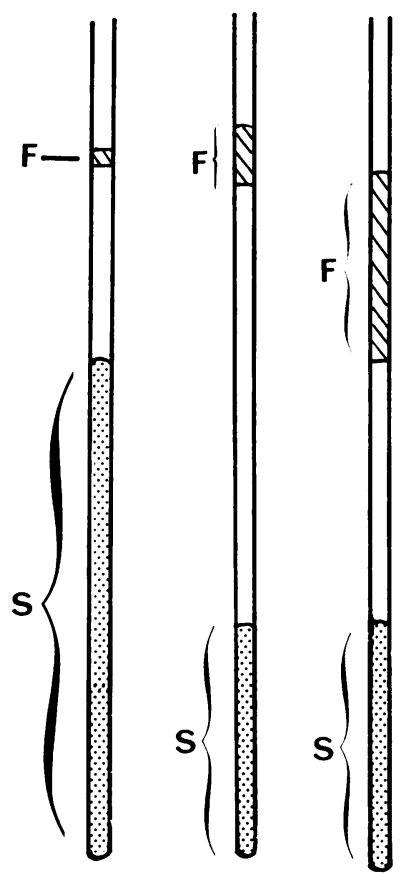

Fig. 1 The steatocrit. Three centrifuged homogenised stool samples with different fat contents. From left to right, steatocrits are $0 \cdot 5 \%, 22 \cdot 3 \%$, and $45 \cdot 2 \% . F$ indicates the fat layer and $S$ the solid layer of the stool.

Clinical material. Ninety-five samples of stool were analysed for steatocrit and stool fat content. Twentyone samples were obtained from 21 healthy term infants on day 5 (12 breast fed and 9 formula fed) with a mean birthweight of $3549 \pm 437 \mathrm{~g}$, and 74 samples from 40 preterm infants of varying postnatal ages ( 33 fed on banked human milk and 7 on formula milk) with a mean birthweight of $1241 \pm 384 \mathrm{~g}$ and a mean gestation of $29 \cdot 6 \pm 3 \cdot 3$ weeks.

\section{Results}

Water content of stool. The mean ( \pm SD) stool water content was $72 \pm 4.9 \%$. The mean stool water content of the 6 babies fed on expressed human milk was $70.9 \%$ (range $62 \cdot 7-77 \cdot 0$ ), and that of the 4 formula-fed infants $73 \cdot 7 \%$ (range $73 \cdot 1-75 \cdot 3$ ). The mean stool water content of the 3 term infants was $74 \cdot 1 \%$ (range $73 \cdot 1-75 \cdot 3$ ), and that from the 7 preterm infants $71 \cdot 1 \%$ (range $62 \cdot 7-77 \cdot 0$ ). There was no relationship, in this small study, between stool water content and type of feed or maturity, thereby excluding stool water content as an important variable in the calculation of the fat content expressed per unit wet weight of the stool.

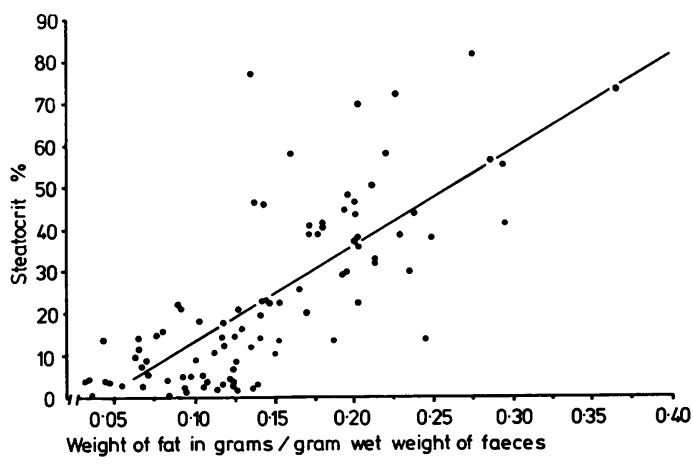

Fig. 2 Correlation between the steatocrit and the stool fat content measured by Sobel's method $(r=0 \cdot 73$, $n=95, P<0.001)$. For 34 steatocrits greater than $25 \%, 31$ had stool fat content $>0.15 \mathrm{gram} / \mathrm{gram}$ wet weight of stool.

Stool fat measurement. Ten steatocrit measurements were performed on each of 2 stool specimens which had widely different fat content. Consecutive measurements on the same specimen of homogenised stool with a high steatocrit value (mean $36 \%$ ) had a coefficient of variation of $2 \%$, the coefficient of variation of the steatocrit on a homogenised stool sample with a low steatocrit value (mean $2 \%$ ) was $12 \%$.

\section{Clinical material}

Fig. 2 shows the correlation between the steatocrit and the measured stool fat content. The fact that the regression line $(r=0.73)$ intersects the $x$-axis rather than passing through the origin probably reflects the lack of precision of the steatocrit on stool samples of very low fat content. For 34 steatocrit values greater than $25 \%, 31(91 \%)$ had stool fat contents $>\mathbf{0} \cdot 15 \mathrm{gram} / \mathrm{gram}$ wet weight of stool. For 61 steatocrits less than $25 \%, 55(90 \%)$ had a stool fat content $<0 \cdot 15 \mathrm{gram} / \mathrm{gram}$ wet weight of stool.

The steatocrits from the 21 term infants were all below $25 \%$; those from the preterm infants varied throughout the range of the results with no constant relationship with gestational or postnatal age, nor was there a relationship with clinical illness. There was no uniform pattern of stool fat concentration in relation to the type of milk ingested, whether it was formula milk, pooled pasteurised human milk, or fresh expressed human milk.

\section{Discussion}

Fat malabsorption is potentially an important cause of poor weight gain among low birthweight and preterm infants. Traditional measurements of stool fat 
require large samples and it often takes as long as a week to complete the analysis. This simple micromethod (steatocrit) requires only $0.5 \mathrm{~g}$ of stool and the whole method takes about 30 minutes.

The steatocrit volumetrically relates the stool fat column to the stool solids column, which are of very different densities and are thus separated in the capillary centrifuge tubes. Although the relationship is imprecise (Fig. 2), it usefully distinguishes those babies with a steatocrit value greater than $25 \%, 91 \%$ of whom had stool fat contents greater than $0 \cdot 15$ gram/gram wet weight of stool, and might arbitrarily be considered to have a degree of fat malabsorption.

The steatocrit estimates stool fat concentration in small, sick infants on whom it is difficult to obtain large enough stool samples for such measurements by standard techniques. Moreover, for babies on formula milks or on banked human milk, the combination of the creamatocrit, the steatocrit, and 24-hour stool weight is a simple guide to daily fat absorption.

We thank Sister $\mathbf{P}$ Lucas, Sister $\mathbf{P}$ Townshend, and the nursing staff for their help with collection of the stools, and Alison Smith for her help with the statistical evaluation of the results.

\section{References \\ 1 Lucas A, Gibbs J A H, Lyster R L J, Baum J D. Creamato- crit: simple clinical technique for estimating fat concentra- tion and energy value of human milk. Br Med J 1978; i: 1018-20. \\ 2 Wybenga D R, James B M, Inkpen A. Lipids. In: Henry R J, Cannon D C, Winkelman J W, eds. Clinical chemistry: principles and techniques, second edition. Hagerstown: Harper \& Row, 1974: 1481-2.}

Correspondence to Dr J D Baum, Department of Paediatrics, John Radcliffe Hospital, Headington, Oxford OX3 9DU.

Received 27 January 1981

\title{
Effects of maternal smoking on fetal growth and nutrition
}

\author{
A $\mathbf{R}$ J BOSLEY, $\mathrm{J}$ R SIBERT, AND R G NEWCOMBE \\ Department of Child Health and Department of Medical Statistics, Welsh National School of Medicine, \\ Cardiff
}

SUMMARY Standard anthropometric measurements were made on 320 term neonates to investigate the influence of smoking on fetal growth and nutrition. Maternal height and triceps skinfold thickness were also measured. Of 320 infants, $126(39 \%)$ were born to mothers who smoked. Maternal triceps skinfold thickness was significantly smaller in smoking mothers. A correlation existed between maternal and infant triceps skinfold thickness. Measurements of infant growth, birthweight, occipito-frontal circumference, and crown-to-heel length were significantly smaller in infants of smoking mothers and remained significantly smaller when corrections were made for maternal triceps skinfold thickness, height, and social class. While these data do not exclude a nutritional mechanism for the effect of maternal smoking on the fetus, the major growth-retarding effects remain after corrections for this. This indicates that the mechanisms are multifactorial. The reduction in occipito-frontal circumference in infants of smoking mothers, and the possible significance of this is stressed.

The adverse effects of maternal smoking on fetal growth as judged by birthweight were first reported by Simpson in 1957. ${ }^{1}$ Numerous subsequent studies have confirmed this observation. Rush ${ }^{2}$ suggested that these adverse effects were mainly because of nutritional differences between mothers who smoked and mothers who did not, but other studies have produced evidence for a direct toxic effect on fetal growth. ${ }^{3}$

This study investigated the relationship between skinfold thickness (SFT) in mothers and anthropometric measurements in their babies related to smoking habits. We hoped this might determine whether a nutritional element exists to mediate the effects of maternal smoking on the fetus.

\section{Methods}

Three hundred and twenty term ( $>37$ weeks' gestation) infants and their mothers were studied. All infants were born in one unit in Cardiff (St David's Hospital) in 1978. Details of the pregnancy and measurements of mother and baby were made by one observer (ARJB), with informed parental consent. Gestation was assessed from menstrual dates and by the clinical method of Parkin et al. ${ }^{4}$

Infant length was measured on the neonatometer (Holtain Ltd). Infant occipito-frontal head circumference (OFC) was measured using a standard paper disposable tape measure. Infant subscapular and 